\title{
Advice to walk after meals is more effective for lowering postprandial glycaemia in type 2 diabetes mellitus than advice that does not specify timing: a randomised crossover study
}

\author{
Andrew N. Reynolds ${ }^{1,2} \cdot$ Jim I. Mann ${ }^{1,2,3} \cdot$ Sheila Williams $^{4} \cdot$ Bernard J. Venn $^{1}$
}

Received: 14 June 2016 / Accepted: 5 August 2016 /Published online: 17 October 2016

(C) Springer-Verlag Berlin Heidelberg 2016

\begin{abstract}
Aims/hypothesis Regular physical activity is a cornerstone of diabetes management. We conducted a study to evaluate whether specifying the timing of walking in relation to meals enhances the benefits conferred by current physical activity guidelines.

Methods A total of 41 adults with type 2 diabetes mellitus (mean $\pm \mathrm{SD}$ age $60 \pm 9.9$ years; mean diabetes duration 10 years) participated in this randomised, crossover study. Randomisation was by a computer-generated protocol. For periods of 2 weeks, advice to walk $30 \mathrm{~min}$ each day was compared with advice to walk for 10 min after each main meal. Both sets of advice met current physical activity guidelines for people with type 2 diabetes mellitus. Physical activity was measured by accelerometry over the full intervention, and glycaemia was measured using continuous glucose monitoring in $5 \mathrm{~min}$ intervals over 7 days. The primary outcome of postprandial glycaemia was assessed during the $3 \mathrm{~h}$ after a meal by the incremental area under the blood glucose curve (iAUC).

Results The iAUC was significantly lower when participants walked after meals compared with on a single daily occasion
\end{abstract}

Jim I. Mann

jim.mann@otago.ac.nz

1 Department of Human Nutrition, University of Otago, P. O. Box 56, Dunedin, Otago 9054, New Zealand

2 Edgar National Centre for Diabetes and Obesity Research, University of Otago, Dunedin, Otago, New Zealand

3 Faculty of Medicine, University of Otago, Dunedin, Otago, New Zealand

4 Department of Preventive and Social Medicine, University of Otago, Dunedin, Otago, New Zealand (ratio of geometric means $0.88,95 \%$ CI $0.78,0.99$ ). The improvement was particularly striking after the evening meal $(0.78,95 \%$ CI $0.67,0.91)$ when the most carbohydrate was consumed and sedentary behaviours were highest. One participant died during the 30 day washout period between interventions, but participation in this study was not judged to contribute to this unfortunate event.

Conclusions/interpretation The benefits relating to physical activity following meals suggest that current guidelines should be amended to specify post-meal activity, particularly when meals contain a substantial amount of carbohydrate.

Trial registration: ACTRN12613000832774

Funding: This study was supported by grants from the University of Otago and the New Zealand Artificial Limb Service. Glycated albumin reagents were provided by Asahi Kasei.

Keywords Hyperglycaemia · Intervention study · Type 2 diabetes mellitus
Abbreviations
CGMS Continuous glucose-monitoring system
iAUC Incremental area under the blood glucose curve

\section{Introduction}

Physical activity guidelines are a cornerstone of advice to prevent and manage type 2 diabetes mellitus [1]. Physical activity has been shown to lower blood glucose levels [2, 3], reduce cardiovascular risk by favourably influencing several metabolic risk factors $[4,5]$ and help reduce body fatness in the many people with type 2 diabetes mellitus who are overweight or obese $[6,7]$. Current physical activity guidelines for 
people with type 2 diabetes mellitus promote at least $150 \mathrm{~min}$ activity per week [8]. Walking or physical activity of similar intensity is advised as it is effective [9, 10], but not associated with an increased cardiovascular risk due to physical exertion in people with established disease [11, 12].

Current guidelines include the option of several blocks of activity during the day in order to aid compliance, but do not specify when physical activity should be taken over the course of the day. Postprandial glycaemia contributes considerably to the overall hyperglycaemia of type 2 diabetes mellitus [13, 14] and is an independent determinant of cardiovascular risk [15, 16]. Studies of a single meal or over a single day suggest that post-meal physical activity can reduce blood glucose levels [17-20]. The current study is the first controlled study in free-living adults with type 2 diabetes mellitus that has aimed to determine whether prescribed walking taken for short periods after meals confers longer-term benefits than walking on a single occasion at any time of the day.

\section{Methods}

Trial design This post-meal walking crossover study involved two interventions in a randomised order, separated by a 30 day washout (Fig. 1). It was conducted between September 2013 and February 2015 at the research clinic of the Department of Human Nutrition, University of Otago, Dunedin, New Zealand. Within each 2 week intervention, participants attended the research clinic on days 1, 7 and 14. On day 1, a fasting blood sample was drawn, measures of anthropometry were taken, the physical activity regimen was prescribed and accelerometers were fitted. On day 7, a continuous glucosemonitoring system (CGMS) was fitted to participant's abdomen, a portable glucometer and testing supplies were provided, and a semi-quantitative 7 day food diary was commenced. On day 14, a fasting blood sample was drawn, measures of anthropometry were taken, the CGMS was removed and the accelerometer and food diary were returned. The same set of procedures was followed during both interventions. The Health and Disability Ethics Committee of the University of Otago approved this study (H13/039), and participants provided written consent before commencing the study.

Participants and eligibility criteria Participants with type 2 diabetes mellitus, and aged 18-75 years, were recruited through general practices, hospital outpatient clinics, the local diabetes society and services that cater for people with chronic diseases. Pregnancy, lactation, and inability or unwillingness to comply with the required physical activity excluded participation. The presence of comorbidities did not exclude participation. A consultant endocrinologist monitored participants for the study duration, but this did not preclude participants visiting their usual healthcare provider, if required.

Randomisation and masking A computer-generated randomisation protocol, undertaken at a separate site, determined the intervention order before the study began. The protocol was undertaken on the advice of a biostatistician (SW), and accessed only after participants were enrolled into the study by the lead author (ANR). The interventions were unmasked.
Fig. 1 Flow chart of participants through the study
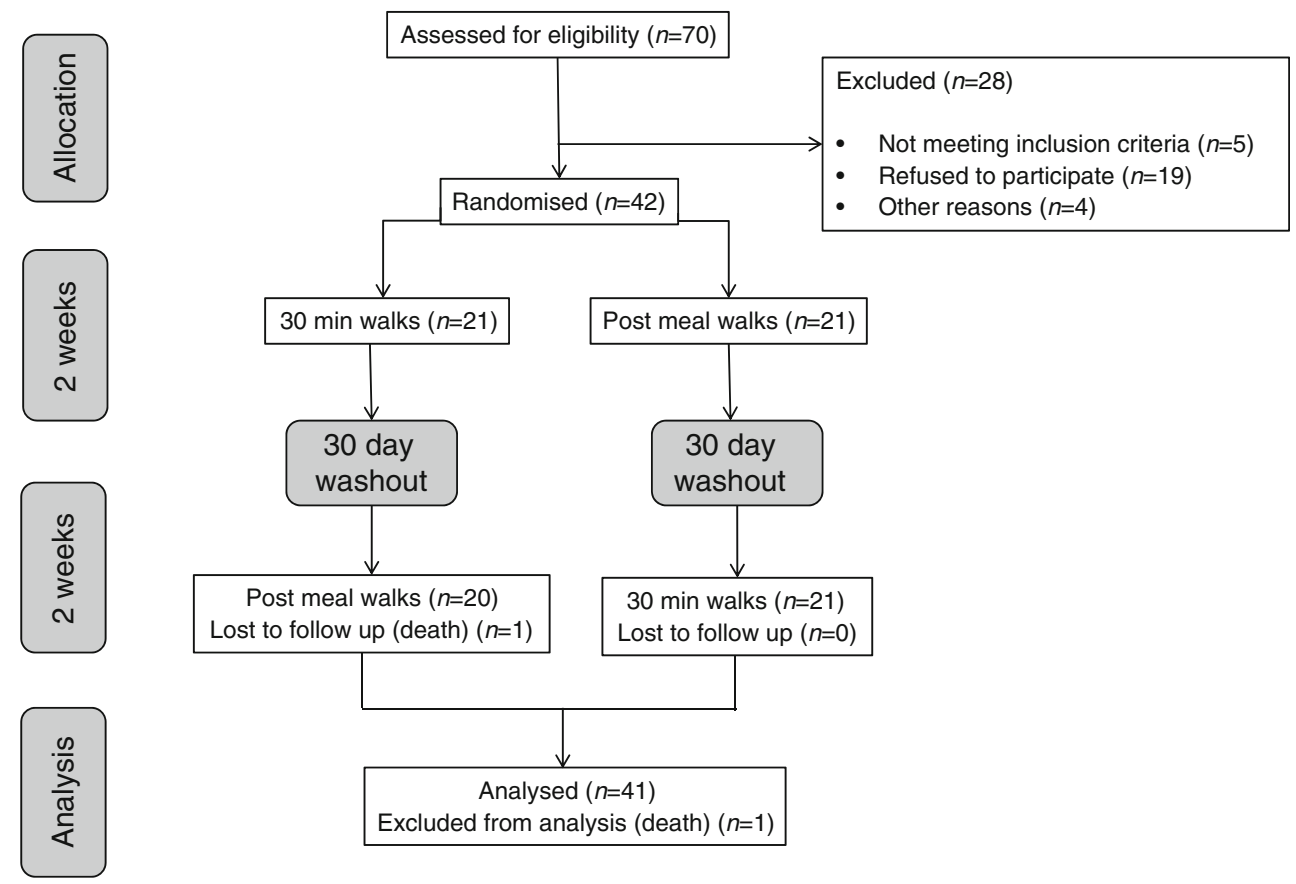
Interventions In order to comply with current physical activity guidelines, both interventions required participants to walk for $30 \mathrm{~min}$ each day. The ' 30 min intervention' involved one walk undertaken at any time of the day. The 'post-meal walking intervention' asked participants to take a 10 min walk after each of the three main meals, starting within 5 min of completing the meal. Participants were advised not to change their diet or lifestyle habits during the study period, beyond complying with the prescribed walking regimen.

Measurements Anthropometric measurements were recorded in duplicate and resting BP was measured three times. The CGMS (iPro, Medtronic, Dublin, Ireland) sampled interstitial glucose every 5 min for 7 days. Each CGMS was calibrated with four capillary glucose tests each day (Optium, Abbott Diabetes, Alameda, CA, USA). Triaxial accelerometers (ActiGraph, Pensacola, FL, USA) were worn during waking hours for the full 14 days of both periods to record activity, including walking and sedentary behaviours. Glucose, $\mathrm{HbA}_{1 \mathrm{c}}$, glycated albumin and lipids were measured in the fasting blood samples.

The primary outcome was postprandial glycaemia, which was assessed during the $3 \mathrm{~h}$ after a meal by the incremental area under the blood glucose curve (iAUC). The iAUC was calculated from meal commencement using the trapezoidal rule, ignoring the area below baseline [21]. Mean glucose over 7 days, the average daily fasting value from 02:00 to 06:00 each morning and measures of glycaemic variability were also recorded. The mean amplitude of glycaemic excursion [22], continuous overall net glycaemic action [23] and glycaemic range (peak minus nadir) were calculated [24], as these measures reflect different aspects of glycaemic variability. Meal and daily fasting responses were omitted from the analysis when more than two consecutive data points were missing within the postprandial or daily fasting exposures. This occurred in $2.4 \%$ of the 1594 meals, and none of the 493 fasting occasions. Interventions were repeated where the CGMS failed while in use.

A predefined range of accelerometer counts was used to determine time spent walking and sedentary time [25]. Both walking and sedentary time are presented as the mean number of minutes within the $60 \mathrm{~min}$ after each meal. Vector magnitude counts/min are provided as an overall measure of daily activity. Total sedentary time is shown as the mean percentage of accelerometer wear time. Dietary data were analysed using food composition tables [26]. Fasting glucose, lipids and $\mathrm{HbA}_{1 \mathrm{c}}$ were analysed on a Cobas C-311 analyser with assay kits (Roche Diagnostics, Risch-Rotkreuz, Switzerland). Glycated albumin was also measured with an assay kit (Asahi Kasei, Tokyo, Japan); the coefficient of variation was $1.3 \%$.

Statistical analysis We based an estimate of the sample size on a power calculation with an alpha of 0.05 and power of
0.80 to detect within-group differences in outcome variables: a clinically relevant $20 \%$ difference in postprandial glycaemia (iAUC) and a 10\% difference in glycated albumin. We needed 28 participants to complete both interventions; however, we over-recruited, with 42 participants enrolling in the study. Data were analysed according to intention to treat. A mixed model, which included a term for order, was used to analyse the data. The results are presented as differences (with $95 \%$ CIs) between the two interventions. The data for iAUC, fasting plasma glucose and triacylglycerols were $\log$ transformed before analysis. Differences in the primary outcome (iAUC) are presented as ratios of the geometric means. Analyses were undertaken using Stata version 13 (StataCorp, College Station, TX, USA), with the statistician blinded to the primary analysis. All results are shown as means $\pm \mathrm{SD}$, unless otherwise stated.

\section{Results}

The flow of participants through the study is shown in Fig. 1. Of the 42 eligible participants randomised, 41 (98\%) completed both intervention periods, exceeding the minimum number required by the power estimate. The participant who did not complete the study died during the 30 day washout period. The mean age of participants was $60 \pm 9.9$ years. Participants were predominately male $(n=26,63 \%)$. Mean $\mathrm{HbA}_{1 \mathrm{c}}$ was 7.5 $\pm 1.42 \%(59 \pm 15.5 \mathrm{mmol} / \mathrm{mol})$ and the mean diabetes duration was $10 \pm 6.6$ years. Most participants were of European descent $(n=33,80 \%)$, while the remainder were of Maori (New Zealand's indigenous people, $n=2)$, Pacific $(n=2)$, Asian $(n=3)$, or Middle Eastern $(n=1)$ descent. The majority $(n=26,63 \%)$ were on oral hypoglycaemic agents, while $27 \%$ $(n=11)$ were on insulin and metformin, and $10 \%(n=4)$ were managed with diet alone. Only two participants (5\%) currently smoked. Before beginning the study the self-reported mean walking time was $14 \mathrm{~min} /$ day [95\% CI 9.4, $18.2 \mathrm{~min} /$ day], with seven participants $(17 \%)$ walking for $30 \mathrm{~min}$ or more each day. Accelerometer wear time did not differ between interventions (10 $\mathrm{min}$ [95\% CI -30, $50 \mathrm{~min}]$ difference in wear time/day).

Table 1 shows the various measures of glycaemia associated with the post-meal and standard 30 min walking regimens. Overall postprandial glycaemia, as measured by the iAUC, was $12 \%(95 \%$ CI $1 \%, 22 \%)$ lower during the post-meal walking period than when following conventional physical activity advice. This difference was largely explained by the highly significant $22 \%$ (95\% CI 9\%, 33\%) difference in iAUC following the evening meal, when the greatest amount of carbohydrate was consumed (Table 2) and when participants tended to be more sedentary (Table 3). Mean blood glucose levels during the $3 \mathrm{~h}$ following the evening meal were significantly lower following post-meal walking $(-0.50 \mathrm{mmol} / \mathrm{l}$ 
Table 1 Measures of glycaemia, adjusted for intervention order, calculated from continuous blood glucose measurements

\begin{tabular}{lcclc}
\hline Measure & $\begin{array}{l}\text { Post-meal } \\
\text { walks }\end{array}$ & $\begin{array}{l}30 \mathrm{~min} \\
\text { walk }\end{array}$ & $\begin{array}{l}\text { Difference }(95 \% \mathrm{CI}) \\
(\text { post-meal walks }-30 \text { min walk) }\end{array}$ & $p$ value \\
\hline All-meal iAUC $\left(\mathrm{mmol} \mathrm{l}^{-1} \mathrm{~min}^{-1}\right)$ & $453 \pm 230$ & $508 \pm 270$ & $0.88(0.78,0.99)$ & 0.031 \\
Breakfast iAUC $\left(\mathrm{mmol} \mathrm{l}^{-1} \mathrm{~min}^{-1}\right)$ & $507 \pm 298$ & $540 \pm 300$ & $0.87(0.72,1.05)$ & 0.153 \\
Lunch iAUC $\left(\mathrm{mmol} \mathrm{l}^{-1} \mathrm{~min}^{-1}\right)$ & $426 \pm 259$ & $444 \pm 264$ & $0.94(0.80,1.10)$ & 0.416 \\
Dinner iAUC $\left(\mathrm{mmol} \mathrm{l}^{-1} \mathrm{~min}^{-1}\right)$ & $424 \pm 240$ & $537 \pm 331$ & $0.78(0.67,0.91)$ & 0.001 \\
FBG (mmol/l) & $8.0 \pm 2.61$ & $7.9 \pm 2.39$ & $0.11(-0.30,0.52)$ & 0.604 \\
Mean BG (mmol/l) & $9.3 \pm 2.55$ & $9.3 \pm 2.46$ & $0.01(-0.29,0.32)$ & 0.932 \\
MAGE (mmol/l) & $4.7 \pm 1.75$ & $5.1 \pm 2.08$ & $-0.42(-0.94,0.10)$ & 0.117 \\
CONGA & $8.4 \pm 2.44$ & $8.4 \pm 2.32$ & $-0.01(-0.31,0.29)$ & 0.944 \\
Glycaemic range (mmol/l) & $12.7 \pm 3.6$ & $13.3 \pm 4.07$ & $-0.60(-1.56,0.36)$ & 0.219 \\
\hline
\end{tabular}

Values are means $\pm \mathrm{SD}$

${ }^{\text {a }}$ Differences are presented as ratios, because the data were log transformed before analysis

${ }^{\mathrm{b}}$ All-meal iAUC is the mean of patient response to all meals recorded within the intervention

BG, blood glucose; CONGA, continuous overall improvement in net glycaemic action; FBG, fasting blood glucose; MAGE, mean amplitude of glycaemic excursion
[95\% CI $-1.0,-0.04 \mathrm{mmol} / \mathrm{l}], p=0.034)$ compared with when timing of walks was not specified.

Amount of carbohydrate consumed did not differ significantly during the two intervention periods, although carbohydrate as a percentage of total energy was slightly greater during the $30 \mathrm{~min}$ walk regimen $(1.8 \%$ [95\% CI $0.4 \%, 3.1 \%$ ], $p=0.008$; Table 2).

Further measures of activity and sedentary time are shown in Table 3. Overall physical activity (counts/minute) was greater during the post-meal walking intervention than when the activity prescription involved a continuous $30 \mathrm{~min}$ walk at any time of the day. Sedentary time was reduced following each meal and walking duration increased after lunch and dinner when post-meal walking rather than a 30 min walk/day was prescribed. Anthropometric, BP and lipid measures before and after each physical activity regimen are shown in Table 4. No significant differences were observed.
Adverse events One participant died during the 30 day washout period of the study. Physical activity was not being undertaken at the time of death. The requirements of this study are not perceived to have contributed to this unfortunate event.

\section{Discussion}

In this study involving patients with type 2 diabetes mellitus, a prescription to walk daily after meals was associated with improvements in postprandial glycaemia when compared with advice to walk for $30 \mathrm{~min} /$ day in a single bout at an unspecified time. Both interventions were consistent with current physical activity guidelines and differed only with regard to the specified time of walking. Although the prescriptions were matched in total walking time, the advice to walk after each main meal resulted in significantly greater
Table 2 Nutrient intake between interventions, adjusted for intervention order

\begin{tabular}{lcclc}
\hline Nutrient & $\begin{array}{l}\text { Post-meal } \\
\text { walks }\end{array}$ & $\begin{array}{l}30 \mathrm{~min} \\
\text { walk }\end{array}$ & $\begin{array}{l}\text { Difference (95\% CI) } \\
\text { (post-meal walks }-30 \text { min walk) }\end{array}$ & $p$ value \\
\hline Energy (kJ) & $8594 \pm 2205$ & $8514 \pm 2109$ & $79(-389,540)$ & 0.753 \\
Protein (\% TE) & $18.3 \pm 2.95$ & $18.1 \pm 2.99$ & $0.2(-0.6,1.1)$ & 0.611 \\
Fat (\% TE) & $40.7 \pm 4.13$ & $39.5 \pm 4.88$ & $1.2(-0.1,2.4)$ & 0.071 \\
Saturated fatty acids (\% TE) & $15.7 \pm 2.73$ & $15.2 \pm 2.71$ & $0.6(-0.1,1.3)$ & 0.111 \\
Dietary fibre (g/day) & $24.1 \pm 5.65$ & $24.1 \pm 5.90$ & $0.0(-1.6,1.6)$ & 0.974 \\
Carbohydrate (\% TE) & $42.8 \pm 5.58$ & $44.6 \pm 6.19$ & $-1.8(-3.1,-0.4)$ & 0.008 \\
Carbohydrate (g/day) & $212.3 \pm 56.44$ & $219.2 \pm 58.95$ & $-6.8(-18.9,5.3)$ & 0.272 \\
$\quad$ Breakfast & $43.0 \pm 21.68$ & $52.3 \pm 26.33$ & $-9.4(-17.5,-1.2)$ & 0.024 \\
$\quad$ Lunch & $49.1 \pm 20.59$ & $42.1 \pm 26.08$ & $6.9(-1.6,15.4)$ & 0.113 \\
$\quad$ Dinner & $66.8 \pm 24.47$ & $59.0 \pm 31.96$ & $8.0(-1.9,17.9)$ & 0.114 \\
\hline
\end{tabular}

Values are means $\pm \mathrm{SD}$

TE, total energy 
Table 3 Measures of physical activity and sedentary time, adjusted for intervention order

\begin{tabular}{lllll}
\hline Variable & $\begin{array}{l}\text { Post-meal } \\
\text { walks }\end{array}$ & $\begin{array}{l}30 \mathrm{~min} \\
\text { walk }\end{array}$ & $\begin{array}{l}\text { Difference (95\% CI) } \\
\text { (post-meal walks }-30 \text { min walk) }\end{array}$ & $p$ value \\
\hline VM (counts/min) & $797.5 \pm 353$ & $707.6 \pm 282$ & $88.35(25.1,152)$ & 0.006 \\
Walking duration (min) & & & & \\
$\quad$ Post breakfast & $12.9 \pm 5.68$ & $11.2 \pm 6.31$ & $1.40(-1.57,4.37)$ & 0.354 \\
$\quad$ Post lunch & $11.9 \pm 4.71$ & $9.1 \pm 5.06$ & $2.74(0.63,4.85)$ & 0.011 \\
$\quad$ Post dinner & $11.4 \pm 5.96$ & $6.8 \pm 3.80$ & $4.51(2.77,6.26)$ & $<0.001$ \\
Total sedentary time (\%) & $72.7 \pm 7.44$ & $73.2 \pm 7.36$ & $-0.45(-0.21,0.01)$ & 0.586 \\
Sedentary time (min) & & & & $<0.001$ \\
$\quad$ Post breakfast & $18.9 \pm 5.87$ & $25.5 \pm 8.55$ & $-6.70(-9.9,-3.5)$ & $<0.001$ \\
$\quad$ Post lunch & $31.8 \pm 9.03$ & $40.5 \pm 7.99$ & $-8.71(-12.2,-5.2)$ & $<0.001$ \\
$\quad$ Post dinner & $40.7 \pm 8.19$ & $45.4 \pm 6.25$ & $-4.70(-7.3,-2.1)$ & \\
\hline
\end{tabular}

Values are means $\pm \mathrm{SD}$

${ }^{\text {a }}$ Mean number of minutes measured in the 60 min after each meal

${ }^{\mathrm{b}}$ Mean percentage of accelerometer wear time per day

$\mathrm{VM}$, vector magnitude counts/min overall activity. The improvement in overall postprandial glycaemia was largely accounted for by lower blood glucose levels after the evening meal, when carbohydrate consumption was high and participants tended to be more sedentary. Accelerometry confirmed that, on average, participants walked for the prescribed duration after meals.

The study was sufficiently powered to generate reliable results and all but one participant completed the study. The CGMS provided an objective, unbiased and sensitive primary endpoint, while accelerometry provided a reliable measure of physical activity. The prescribed level of activity was modest, so the results may be regarded applicable to a wide group of patients with type 2 diabetes mellitus who are sufficiently motivated to undertake physical activity. A limitation of the study is that the interventions were of insufficient duration to confirm that the improvement in postprandial glycaemia translated into an improvement in overall glycaemic control.
Nevertheless, postprandial glycaemia is an important determinant of glycaemic control in its own right $[13,14]$. It would be useful in future studies to include biochemical or cell-based measurements that might help to explain the mechanisms by which increased walking after meals can reduce blood glucose levels [1].

Our study is the first controlled study in patients with type 2 diabetes mellitus to examine the effects of post-meal activity on postprandial glycaemia in free-living individuals, and the first to have continued over a period longer than 2 days. Several previous studies have shown reduced postprandial glycaemia when a meal has been followed by physical activity compared with remaining sedentary [17-20, 27-30]. These studies have typically included eight to 12 participants observed in a controlled setting with meals provided. Previous studies have varied in the frequency at which blood glucose was measured, although this has usually been at

Table 4 Anthropometric, BP and lipid measures, adjusted for intervention order

\begin{tabular}{|c|c|c|c|c|c|c|}
\hline \multirow[t]{2}{*}{ Measure } & \multicolumn{2}{|c|}{ Post-meal walks } & \multicolumn{2}{|l|}{30 min walk } & \multirow{2}{*}{$\begin{array}{l}\text { Difference }(95 \% \mathrm{CI}) \\
\text { (post-meal walks }-30 \text { min walk) }\end{array}$} & \multirow[t]{2}{*}{$p$ value } \\
\hline & Pre & Post & Pre & Post & & \\
\hline BMI $\left(\mathrm{kg} / \mathrm{m}^{2}\right)$ & $31.7 \pm 3.84$ & $31.5 \pm 3.87$ & $31.9 \pm 4.03$ & $31.6 \pm 4.04$ & $0.2(-0.1,0.5)$ & 0.221 \\
\hline Waist circumference $(\mathrm{cm})$ & $107.8 \pm 10.9$ & $107.1 \pm 13.5$ & $108.5 \pm 11.0$ & $108.4 \pm 11.2$ & $-0.7(-3.5,2.2)$ & 0.644 \\
\hline Systolic BP (mmHg) & $147 \pm 16.5$ & $143 \pm 14.1$ & $146 \pm 16.2$ & $142 \pm 15.5$ & $0.7(-4.0,5.5)$ & 0.762 \\
\hline Glycated albumin (\%) & $18.1 \pm 4.49$ & $17.6 \pm 4.34$ & $18.0 \pm 4.26$ & $17.2 \pm 4.14$ & $0.3(-0.1,0.8)$ & 0.175 \\
\hline Fasting plasma glucose $(\mathrm{mmol} / \mathrm{l})^{\mathrm{a}}$ & $9.4 \pm 3.02$ & $9.3 \pm 3.01$ & $8.9 \pm 2.57$ & $8.4 \pm 2.24$ & $0.1(0.0,0.2)$ & 0.115 \\
\hline Total cholesterol (mmol/l) & $4.4 \pm 1.23$ & $4.2 \pm 1.13$ & $4.3 \pm 1.09$ & $4.2 \pm 1.29$ & $-0.1(-0.3,0.2)$ & 0.629 \\
\hline HDL-cholesterol (mmol/1) & $1.5 \pm 0.50$ & $1.4 \pm 0.53$ & $1.4 \pm 0.50$ & $1.3 \pm 0.45$ & $0.0(-0.2,0.1)$ & 0.956 \\
\hline Triacylglycerols $(\mathrm{mmol} / \mathrm{l})^{\mathrm{a}}$ & $1.4 \pm 0.85$ & $1.4 \pm 0.67$ & $1.4 \pm 0.60$ & $1.3 \pm 0.51$ & $0.1(0.0,0.2)$ & 0.109 \\
\hline
\end{tabular}

Values are mean \pm SD unless stated otherwise

${ }^{a}$ Fasting plasma glucose and triacylglycerols were log transformed 
30 min intervals following a single meal. Studies have also examined the effect of individual meals, whereas using a CGMS enabled us to measure glucose at $5 \mathrm{~min}$ intervals over a 7 day period. Furthermore, additional previous studies have examined the effects of physical activity, but not specified the timing of activity in relation to meals [31, 32].

Reduction of postprandial glucose is regarded as an important target in the management of type 2 diabetes mellitus, given its independent contribution to glycaemic control and cardiovascular risk $[13,15,16]$. Postprandial physical activity may avoid the need for an increased total insulin dose or additional mealtime insulin injections that might otherwise have been prescribed to lower glucose levels after eating. An increase in insulin dose might, in turn, be associated with weight gain in patients with type 2 diabetes mellitus, many of whom are already overweight or obese. Our findings provide evidence for the amendment of guidelines to specifically include post-meal activity, especially after meals containing a substantial amount of carbohydrate.

In conclusion, while the existing data are arguably sufficient to recommend at least $10 \mathrm{~min}$ of walking after meals, additional research would enable this advice to be further refined. Many patients with type 2 diabetes mellitus may not be able or willing to undertake physical activity of greater intensity. However, it would be helpful to know whether a longer walk would produce a greater benefit when undertaken after the meal providing the highest amount of carbohydrate each day. A study of longer duration would demonstrate if the reduction in postprandial blood glucose levels translates into an overall improvement in glycaemic control. Perhaps most important of all would be research aimed at identifying means of achieving adherence with advice to increase physical activity.

Acknowledgements We thank all participants of this study. We also thank C. Higgs and the Dunedin Community Exercise Programme, M. Hanton and the Mornington Health Centre, N. Wedlock and Diabetes Otago, general practices, and the Dunedin Hospital diabetes clinic, who assisted with recruitment; A. Duncan and M. Harper (Diabetes Laboratory, Department of Human Nutrition, University of Otago); G. Paterson (Department of Human Nutrition, University of Otago) for drawing the majority of venous blood samples and her work as research nurse; S. Langford (consulting engineer) for his contribution to data tabulation; and Z. Zhang (Department of Human Nutrition, University of Otago) for the initial concept.

Funding This study was supported by grants from the University of Otago and the New Zealand Artificial Limb Service. Glycated albumin reagents were provided by Asahi Kasei. The funders of the study had no role in the study design, data collection, data analysis, data interpretation or writing of the report.

Duality of interest All of authors have completed the ICMJE Unified Competing Interests form (available on request from the corresponding author). The authors declare that there is no duality of interest associated with this manuscript
Contribution statement ANR contributed to the study design, and was responsible for the study's implementation, conduct and monitoring. ANR undertook the analyses and interpretation of results, wrote the first draft and was responsible for editing the manuscript. JIM contributed to the study design and monitoring, and provided expertise and guidance over the study duration. JIM was involved with the interpretation of results and was responsible for editing the manuscript. SW contributed to the study design and undertook the analyses. SW contributed to the interpretation of the study and was responsible for editing the manuscript. BJV developed the initial concept, contributed to the study design and was responsible for editing the manuscript. All authors approved the final draft of the manuscript. JIM, as corresponding author, had full access to the data in the study and had the final decision to submit the paper for publication.

\section{References}

1. Sigal RJ, Kenny GP, Wasserman DH, Castaneda-Sceppa C (2004) Physical activity/exercise and type 2 diabetes. Diabetes Care 27: 2518-2539

2. Sigal RJ, Kenny GP, Wasserman DH, Castaneda-Sceppa C, White RD (2006) Physical activity/exercise and type 2 diabetes: a consensus statement from the American Diabetes Association. Diabetes Care 29:1433-1438

3. Wannamethee SG, Shaper AG, Alberti KG (2000) Physical activity, metabolic factors, and the incidence of coronary heart disease and type 2 diabetes. Arch Intern Med 160:2108-2116

4. Thompson PD, Buchner D, Piña IL et al (2003) Exercise and physical activity in the prevention and treatment of atherosclerotic cardiovascular disease: a statement from the Council on Clinical Cardiology (Subcommittee on Exercise, Rehabilitation, and Prevention) and the Council on Nutrition, Physical Activity, and Metabolism (Subcommittee on Physical Activity). Circulation 107:3109-3116

5. Bassuk SS, Manson JE (2005) Epidemiological evidence for the role of physical activity in reducing risk of type 2 diabetes and cardiovascular disease. J Appl Physiol 99:1193-1204

6. Knowler WC, Barrett-Connor E, Fowler SE et al (2002) Reduction in the incidence of type 2 diabetes with lifestyle intervention or metformin. N Engl J Med 346:393-403

7. Wadden TA, West DS, Neiberg RH et al (2009) One-year weight losses in the Look AHEAD Study: factors associated with success. Obesity 17:713-722

8. Mendes R, Sousa N, Almeida A et al (2015) Exercise prescription for patients with type 2 diabetes - a synthesis of international recommendations: narrative review. Br J Sports Med. doi:10.1136 /bjsports-2015-094895

9. Hu FB, Sigal RJ, Rich-Edwards JW et al (1999) Walking compared with vigorous physical activity and risk of type 2 diabetes in women: a prospective study. JAMA 282:1433-1439

10. Lynch J, Helmrich SP, Lakka TA et al (1996) Moderately intense physical activities and high levels of cardiorespiratory fitness reduce the risk of non-insulin-dependent diabetes mellitus in middle-aged men. Arch Intern Med 156:1307-1314

11. Stamler J, Vaccaro O, Neaton JD, Wentworth D (1993) Diabetes, other risk factors, and 12-yr cardiovascular mortality for men screened in the Multiple Risk Factor Intervention Trial. Diabetes Care 16:434-444

12. Kannel WB, McGee DL (1979) Diabetes and cardiovascular disease: the Framingham study. JAMA 241:2035-2038 
13. Monnier L, Lapinski H, Colette C (2003) Contributions of fasting and postprandial plasma glucose increments to the overall diurnal hyperglycemia of type 2 diabetic patients variations with increasing levels of HbA1c. Diabetes Care 26:881-885

14. Woerle HJ, Neumann C, Zschau S et al (2007) Impact of fasting and postprandial glycemia on overall glycemic control in type 2 diabetes: importance of postprandial glycemia to achieve target $\mathrm{HbA1c}$ levels. Diabetes Res Clin Pract 77:280-285

15. Bonora E, Muggeo M (2001) Postprandial blood glucose as a risk factor for cardiovascular disease in type II diabetes: the epidemiological evidence. Diabetologia 44:2107-2114

16. Bruno A, Biller J, Adams H et al (1999) Acute blood glucose level and outcome from ischemic stroke. Neurology 52:280-280

17. Heden TD, Winn NC, Mari A et al (2015) Postdinner resistance exercise improves postprandial risk factors more effectively than predinner resistance exercise in patients with type 2 diabetes. J Appl Physiol 118:624-634

18. Larsen J, Dela F, Kjær M, Galbo H (1997) The effect of moderate exercise on postprandial glucose homeostasis in NIDDM patients. Diabetologia 40:447-453

19. Larsen J, Dela F, Madsbad S, Galbo H (1999) The effect of intense exercise on postprandial glucose homeostasis in type II diabetic patients. Diabetologia 42:1282-1292

20. van Dijk J-W, Venema M, van Mechelen W, Stehouwer CD, Hartgens F, van Loon LJ (2013) Effect of moderate-intensity exercise versus activities of daily living on 24-hour blood glucose homeostasis in male patients with type 2 diabetes. Diabetes Care 36: $3448-3453$

21. Matthews J, Altman DG, Campbell M, Royston P (1990) Analysis of serial measurements in medical research. BMJ 300:230-235

22. Molnar GD, Rosevear JW, Ackerman E, Gatewood LC, Taylor WF (1970) Mean amplitude of glycemic excursions, a measure of diabetic instability. Diabetes 19:644-655
23. McDonnell C, Donath S, Vidmar S, Werther G, Cameron F (2005) A novel approach to continuous glucose analysis utilizing glycemic variation. Diabetes Technol Ther 7:253-263

24. Hill NR, Oliver NS, Choudhary P, Levy JC, Hindmarsh P, Matthews DR (2011) Normal reference range for mean tissue glucose and glycemic variability derived from continuous glucose monitoring for subjects without diabetes in different ethnic groups. Diabetes Technol Ther 13:921-928

25. Freedson PS, Melanson E, Sirard J (1998) Calibration of the Computer Science and Applications, Inc. accelerometer. Med Sci Sports Exerc 30:777-781

26. Athar N, Spriggs TW, Liu P (1999) The concise New Zealand food composition tables. New Zealand Institute for Crop \& Food Research Limited, Palmerston North, New Zealand

27. Colberg SR, Grieco CR, Somma CT (2014) Exercise effects on postprandial glycemia, mood, and sympathovagal balance in type 2 diabetes. J Am Med Dir Assoc 15:261-266

28. Manders R, van Dijk J, van Loon L (2010) Low-intensity exercise reduces the prevalence of hyperglycemia in type 2 diabetes. Med Sci Sports Exerc 42:219-225

29. Tobin L, Kiens B, Galbo H (2008) The effect of exercise on postprandial lipidemia in type 2 diabetic patients. Eur J Appl Physiol 102:361-370

30. Colberg SR, Zarrabi L, Bennington L et al (2009) Postprandial walking is better for lowering the glycemic effect of dinner than pre-dinner exercise in type 2 diabetic individuals. J Am Med Dir Assoc 10:394-397

31. Mikus CR, Oberlin DJ, Libla J, Boyle LJ, Thyfault JP (2012) Glycaemic control is improved by 7 days of aerobic exercise training in patients with type 2 diabetes. Diabetologia 55:1417-1423

32. Karstoft K, Winding K, Knudsen SH et al (2013) The effects of free-living interval-walking training on glycemic control, body composition, and physical fitness in type 2 diabetic patients. A randomized, controlled trial. Diabetes Care 36:228-236 